\title{
Design and Evaluation of Hydrophilic Matrix System Containing Polyethylene Oxides for the Zero-Order Controlled Delivery of Water-Insoluble Drugs
}

\author{
Lijie Wang, ${ }^{1}$ Kai Chen, ${ }^{1}$ Haoyang Wen, ${ }^{1}$ Defang Ouyang, ${ }^{2}$ Xue Li, ${ }^{1}$ Yunyun Gao, \\ Weisan Pan, ${ }^{1}$ and Xinggang Yang ${ }^{1,3,4}$
}

Received 14 December 2015; accepted 4 February 2016; published online 16 February 2016

\begin{abstract}
The aim of this study was to design a polyethylene oxide (PEO) binary hydrophilic matrix controlled system and investigate the most important influence(s) on the in vitro water-insoluble drug release behavior of this controlled system. Direct-compressed PEO binary matrix tablets were obtained from a variety of low viscosity hydrophilic materials as a sustained agent, using anhydrous drugs as a model drug. Water uptake rate, swelling rate, and erosion rate of matrices were investigated for the evaluation of the PEO hydrophilic matrix systems. The effect of the dose, the solubility of water-insoluble drug, and the rheology of polymers on in vitro release were also discussed. Based on the in vitro release kinetics study, three optimized PEO binary matrices were selected for further research. And, these PEO binary matrices had shown the similar release behavior that had been evaluated by the similarity factor $f_{2}$. Further study indicated that they had identical hydration, swelling, and erosion rate. Moreover, rheology study exhibited the similar rheological equation of Herschel-Bulkley and their viscosity was also within the same magnitude. Therefore, viscosity plays the most important role to control drug release compared to other factors in PEO binary matrix system. This research provides fundamental understanding of in vitro drug release of PEO binary hydrophilic matrix tablets and helps pharmaceutical workers to develop a hydrophilic controlled system, which will effectively shorten the process of formulation development by reducing trial-and-error.
\end{abstract}

KEY WORDS: hydrophilic matrix system; polyethylene oxides; water insoluble drug; in vitro release; viscosity.

\section{INTRODUCTION}

Today, oral extended-release (ER) and controlledrelease (CR) formulations become more and more important because of their unique advantages, such as better patient compliance, less fluctuation of blood drug concentrations, reduced side effects, and increased safety $(1,2)$. Among various ER/CR dosage systems, the most famous one is the hydrophilic matrix tablet (3-5). Hydrophilic matrix tablets are usually assembled by hydrophilic polymer and/or other excipients with homogeneous dispersion of drug. The mechanism of drug release from the matrix is mainly through the polymer swelling and dissolution $(6,7)$. Thus, the hydrophilic polymer in the formulations is very important to control drug release (8). There are two mechanisms to affect the drug release: one

\footnotetext{
${ }^{1}$ Department of Pharmaceutics, School of Pharmacy, Shenyang Pharmaceutical University, Shenyang, 110000, China.

${ }^{2}$ State Key Laboratory of Quality Research in Chinese Medicine, Institute of Chinese Medical Sciences (ICMS), University of Macau, Macau, China.

${ }^{3}$ Shenyang Pharmaceutical University, No.103, Wenhua Road, Shenyang, 110016, China.

${ }^{4}$ To whom correspondence should be addressed. (e-mail: yangxg123@163.com)
}

is the water absorption and then drug diffusion through gel layer; the other is water absorption and then drug release by gel erosion. Water uptake is particularly important to both mechanisms. After water absorption reaches a critical value, a gel layer will be formed at the matrix surface. In the gel layer, folding polymer chains gradually open and dissolve, then the process gradually moves to the dry core of the matrix until the tablet core completely disappears. Drug solubility is an important factor to this process. For water-soluble drugs, drug release is mainly influenced by the water and drug diffusion, while for water-insoluble drugs are more likely controlled by the swelling and erosion rate of the matrices (9-11).

In addition to drug solubility, drug release can also be modified by the polymer type and polymer viscosity (4). The Stokes-Einstein equation and Wilke correlation has shown the relationship between the viscosity of polymers and the drug overall release rate (12). Polymer erosion is determined by polymer concentration at the gel layer. One important parameter is the critical concentration $\left(C_{\text {crit }}\right)$. The $C_{\text {crit }}$ is the lowest polymer concentration in the formulations at which the polymer chains can withstand the surrounding share forces without being released (13). For a linear polymer, the $C_{c r i t}$ is determined only by the molecular weights or viscosity grades of the polymers. One of widely used hydrophilic linear polymer is polyethylene oxide (PEO) because of its good compatibility, 
mobility, and chemical stability (14). When PEOs contact with water, because of their high water affinity, they can form strong hydrogen bonding force with the water. With the diffusion of water into the matrix, the force becomes stronger and the molecular chains become swelling and erosion $(15,16)$. Although many scientists have studied the extended release hydrophilic matrices, fewer results demonstrated constant zero-order release in hydrophilic matrix tablets for waterinsoluble drugs (17-20).

The purpose of this study was to design a PEO binary hydrophilic matrix controlled system and investigate the key influencing factors of PEO hydrophilic matrix systems on in vitro release rate for poorly soluble drugs. PEO was chosen as the main polymer added in the hydrophilic matrix systems and other low-viscosity hydrophilic materials were also added in this system to change the drug release rate. Different parameters of these matrix systems should be characterized, including the matrix hydration, matrix swelling, erosion rate, and the rheology of hydrophilic polymer so that the controlled release mechanism could be well elucidated. These PEO binary hydrophilic systems could provide a good zero-order drug release and offer us an effective guidance to design the PEO matrix tablet formulation.

\section{MATERIALS AND METHODS}

The following materials were used in the manufacture of the matrix tablets: gliclazide (GLZ, TianJin, China), nimodipine (NIM, WuHan, China), and theophylline (TPL, WuHan, China); WSRN 301 and PEON 80 were gained from Dow Chemical Co. (Piscataway Township, NJ); polyethylene glycol 4000 (PEG 4000, TianJin, China); poloxamer 188 (F68) was provided by BASF Co. (Guangzhou, China); microcrystalline cellulose was obtained from (MCC, AnHui, China); and magnesium stearate was supplied from (MS, Shanghai, China). Other chemicals were of analytical grade.

\section{Study of GLZ Matrix Tablets}

The PEO binary hydrophilic matrix system was needed to further study the critical factors that influence the waterinsoluble drug release as the zero-order kinetics. In this section, GLZ was chosen as a model drug to obtain the optimized zero-order release formulations.

\section{Preparation of GLZ Matrix Tablets}

Seven formulations of GLZ (30 mg) matrix tablets were prepared to adopt three different kinds of the small molecular material (PEON 80, F68, and PEG 4000). Table I showed the composition of the studied formulations. All the materials were blended for $10 \mathrm{~min}$ in a mortar with the exception of MS. After addition of MS, the mixing procedure was continued for another $5 \mathrm{~min}$. Tablets $(273 \pm 3 \mathrm{mg})$ of each group were made by direct compression with a single-punch tablet press machine (SOP-TDP-5, Shanghai, China) using a 9-mm diameter die and manual feeding. The compression force has been measured by TBH 20 (ERWEKA, Germany) to ensure that these matrix tablets' hardness was in $9-10 \mathrm{kP}$.

\section{In Vitro GLZ Release Studies}

Release experiments (six tablets) were studied in an automatic paddle with USP apparatus type 2 by employing ZRCD6-B (Shanghai Huanghai Medicine Checking Instrument Co., Ltd., Shanghai, China) dissolution tester with a rotation speed of $100 \mathrm{rpm}$ at $37 \pm 0.5^{\circ} \mathrm{C}$. The matrix tablets were locked in the basket which laid the bottom of the cup to avoid them adhering to the cup. The dissolution media $(1000 \mathrm{ml})$ were phosphate-buffered solution (pH 7.4). A sample $(5 \mathrm{ml})$ of the solution was withdrawn from the dissolution apparatus at specific time intervals (1, 2, 4, 6, 8, 10, and $12 \mathrm{~h}$ ), and then analyzed for drug release at a wavelength of $226 \mathrm{~nm}$ using a UV-9100 (Ruili, China). The cumulative percentages of the drug released were analyzed according to zero-order kinetics, first-order kinetics, and Korsmeyer-Peppas (21) equations.

Zero-order : $M t / M_{\infty}=K t$

First-order $: \ln (1-M t / M \infty)=-K t$

Korsmeyer-Peppas : $M t / M \infty=K t^{n}$

Where $M_{t}$ is the fractional drug release at time $t, M_{\infty}$ is the amount of drug loading at infinite time; $K$ is a kinetic constant that measures the release rate; and $n$ is the diffusion exponent characteristic that depends on the release mechanism and the geometry of the system. In Eq. (3), for a cylindrical matrix, $0.89<n<1.0$ indicates a zero-order release,

Table I. Composition of the Different Formulations of GLZ Prepared

\begin{tabular}{|c|c|c|c|c|c|c|c|}
\hline Formulation & GLZ & WSRN 301 & PEON 80 & PL 188 & PEG4000 & $\mathrm{MCC}$ & MS \\
\hline G-1 & 30 & 0 & 120 & - & - & 120 & 3 \\
\hline G-2 & 30 & 60 & 60 & - & - & 120 & 3 \\
\hline G-3 & 30 & 0 & - & 120 & - & 120 & 3 \\
\hline G-4 & 30 & 60 & - & 60 & - & 120 & 3 \\
\hline G-5 & 30 & 0 & - & - & 120 & 120 & 3 \\
\hline G-6 & 30 & 60 & - & - & 60 & 120 & 3 \\
\hline G-7 & 30 & 120 & - & - & - & 120 & 3 \\
\hline
\end{tabular}


Table II. Composition of the Different Doses Formulations of GLZ Prepared

\begin{tabular}{|c|c|c|c|c|c|c|c|}
\hline Formulation & GLZ & WSRN 301 & PEON 80 & F68 & PEG4000 & $\mathrm{MCC}$ & MS \\
\hline $\mathrm{G}-1-30$ & 30 & 60 & 60 & - & - & 120 & 3 \\
\hline G-2-60 & 60 & 60 & 60 & - & - & 90 & 3 \\
\hline G-3-90 & 90 & 60 & 60 & - & - & 60 & 3 \\
\hline G-4-120 & 120 & 60 & 60 & - & - & 30 & 3 \\
\hline $\mathrm{G}-5-30$ & 30 & 60 & - & 60 & - & 120 & 3 \\
\hline G-6-60 & 60 & 60 & - & 60 & - & 90 & 3 \\
\hline G-7-90 & 90 & 60 & - & 60 & - & 60 & 3 \\
\hline G-8-120 & 120 & 60 & - & 60 & - & 30 & 3 \\
\hline G-9-30 & 30 & 60 & - & - & 60 & 120 & 3 \\
\hline G-10-60 & 60 & 60 & - & - & 60 & 90 & 3 \\
\hline G-11-90 & 90 & 60 & - & - & 60 & 60 & 3 \\
\hline G-12-120 & 120 & 60 & - & - & 60 & 30 & 3 \\
\hline
\end{tabular}

The model drug and other excipients in the prescription are in milligram dosage level

while $0.45<n<0.89$ states anomalous release kinetics. The release profiles of these formulations were compared by the similarity factor $f_{2}(22,23)$. The values of $f_{2}$ were calculated by Eq. (4):

$f_{2}=50 \times \lg \left\{\left[1+(1 / T) \sum_{i=1}^{T}\left(\bar{x}_{t i}-\bar{x}_{r i}\right)^{2}\right]^{-1 / 2} \times 100\right\}$

The similarity factor $f_{2}$ was recommended by the FDA to evaluate the similarity of the in vitro dissolution curves. Profiles were considered to be similar if $50<f_{2}<100$. And, the $f_{2}$ values were far more than 50 which indicate drug release profiles at the same level or have the same release kinetics. The closer the $f_{2}$ value is to 100 , the more similar or identical the release profiles are.

Based on studying GLZ formulations, the optimized PEO binary matrix systems were achieved and then their characteristics were investigated.

\section{Study on the Applicability of the PEO Hydrophilic Matrix Systems}

In order for the applicability of these systems to be used in designing controlled drug delivery formulation, the study on the range of dose and drug solubility was performed in the following experiments. In this section, the optimized binary matrix system was chosen as the model matrix; different doses of GLZ and different solubility drug, such as NIM and TPL, were investigated.

\section{The Range of the Dose of Water-Insoluble Drug in These Systems}

According to the above experimental methods, the different doses of matrix tablets were prepared for further study. Table II shows different GLZ dose $(30,60,90,120 \mathrm{mg})$ formulations. The in vitro release profiles at different time points were calculated. And, zero-order, first-order, and KorsmeyerPeppas equations were used to analyze drug release profiles. The similarity factor $f_{2}$ was used to compare the similarity of these drug release profiles.

\section{The Range of the Solubility of the Insoluble Drug in These Systems}

In this study, two groups of PEO matrix tablet formulations were investigated using the method of the GLZ preparation and the composition of this study formulation shown in Table III. To find out the range of the drug solubility, the lowsolubility drug NIM $\left(2.29 \mu \mathrm{g} / \mathrm{ml}\right.$, at $\left.37 \pm 0.1^{\circ} \mathrm{C}\right)(24)$ and highsolubility TPL $\left(8.3 \mathrm{mg} / \mathrm{ml}\right.$, at $\left.25 \pm 0.1^{\circ} \mathrm{C}\right)(25)$ were chosen as model drugs.

The whole process of preparation of NIM matrices must be protected from light because the degradation of NIM will occur easily in the solution (26). This dissolution study was carried out in a 900-ml phosphate-buffered solution (PBS, $\mathrm{pH}$ 7.4 ) including $0.5 \%$ sodium dodecyl sulfate (SDS). And, the water bath condition was set up to $37 \pm 0.5^{\circ} \mathrm{C}$ and the dissolution medium was sequentially stirred at $100 \mathrm{rpm}$. In predecided interval $(1,2,4,6,8,10$, and $12 \mathrm{~h}), 5 \mathrm{ml}$ of dissolution medium was taken out and then measured under a UV-9100 spectrometer at $360 \mathrm{~nm}$.

Table III. Composition of the Formulations of NIM and TPL Prepared

\begin{tabular}{|c|c|c|c|c|c|c|c|}
\hline Formulation & & WSRN 301 & PEON 80 & F68 & PEG 4000 & $\mathrm{MCC}$ & MS \\
\hline \multirow[t]{3}{*}{ NIM (60mg) } & N-1 & 60 & 60 & - & - & 90 & 3 \\
\hline & $\mathrm{N}-2$ & 60 & - & 60 & - & 90 & 3 \\
\hline & $\mathrm{N}-3$ & 60 & - & - & 60 & 90 & 3 \\
\hline \multirow[t]{3}{*}{ TPL (100mg) } & $\mathrm{T}-1$ & 60 & 60 & - & - & 50 & 3 \\
\hline & $\mathrm{T}-2$ & 60 & - & 60 & - & 50 & 3 \\
\hline & $\mathrm{T}-3$ & 60 & - & - & 60 & 50 & 3 \\
\hline
\end{tabular}


Table IV. The Kinetic Parameters of Formulations

\begin{tabular}{|c|c|c|c|c|c|c|c|c|c|c|}
\hline \multirow[t]{2}{*}{ Formulation } & \multicolumn{3}{|c|}{ Zero-order } & \multicolumn{3}{|c|}{ First-order } & \multicolumn{3}{|c|}{ Korsmeyer-Peppas } & \multirow[t]{2}{*}{$n$} \\
\hline & $r^{2}$ & $\mathrm{~T}_{50}(\mathrm{~h})$ & $K\left(\mathrm{~h}^{-1}\right)$ & $r^{2}$ & $\mathrm{~T}_{50}(\mathrm{~h})$ & $K\left(\mathrm{~h}^{-1}\right)$ & $r^{2}$ & $\mathrm{~T}_{50}(\mathrm{~h})$ & $K\left(\mathrm{~h}^{-1}\right)$ & \\
\hline G-1 & 0.9565 & 8.60 & 0.10 & 0.9557 & 11.86 & 0.001 & 0.9834 & 29.78 & 0.16 & 0.76 \\
\hline G-2 & 0.9989 & 9.65 & 0.09 & 0.9989 & 13.32 & 0.001 & 0.9990 & 10.65 & 0.09 & 0.98 \\
\hline G-3 & 0.9248 & 8.28 & 0.10 & 0.9237 & 11.41 & 0.001 & 0.9721 & 43.21 & 0.19 & 0.71 \\
\hline G-4 & 0.9951 & 9.55 & 0.09 & 0.9949 & 13.19 & 0.001 & 0.9973 & 13.74 & 0.10 & 0.92 \\
\hline G-5 & 0.9374 & 9.51 & 0.09 & 0.9365 & 13.13 & 0.001 & 0.9943 & 67.73 & 0.18 & 0.68 \\
\hline G-6 & 0.9957 & 9.38 & 0.09 & 0.9955 & 12.95 & 0.001 & 0.9994 & 14.89 & 0.11 & 0.90 \\
\hline G-7 & 0.9769 & 15.07 & 0.06 & 0.9767 & 20.84 & 0.001 & 0.9913 & 43.75 & 0.08 & 0.81 \\
\hline
\end{tabular}

$r^{2}$ correlation coefficient, $T_{50}$ time to release $50 \%$ of drug, $K$ dissolution rate of constant, $n$ the diffusion exponent characteristic

In vitro dissolution tests of TPL matrices were employed in 1000 -ml distilled water at $37 \pm 0.5^{\circ} \mathrm{C}$, with locking in the basket and the paddle speed of $100 \mathrm{rpm}$. Sample of TPL solution $(5 \mathrm{ml})$ was taken out at the interval $(1,2,4,6,8,10$, and $12 \mathrm{~h}$ ). The concentration of TPL was measured under a UV-9100 spectrometer at $236 \mathrm{~nm}$.

NIM and TLP release profiles were analyzed according to the zero-order kinetics, first-order kinetics, and Korsmeyer-Peppas equations. In addition, the similarity of these drug release profiles was assessed by using the similarity factor $f_{2}$.

\section{Study of the Characteristics of this Hydrophilic Matrix System}

In order to further explain the mechanism of this system control's drug release, the water uptake, swelling rate, and erosion rate of PEO binary matrix tablets should be studied.

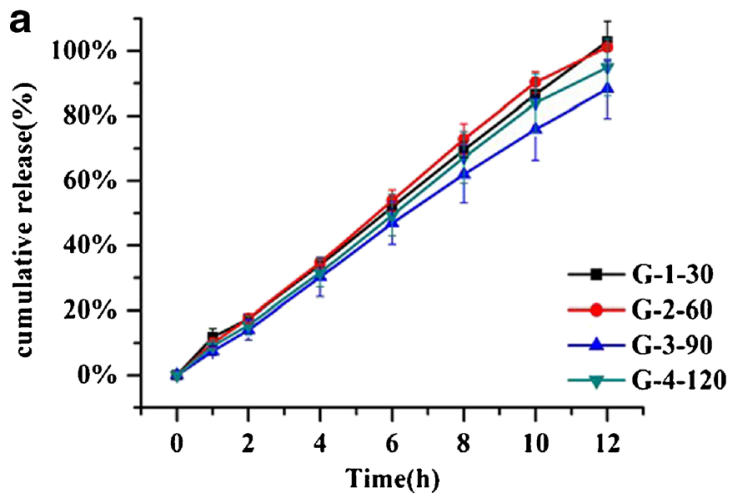

And, the effect of the $\mathrm{pH}$ of dissolution medium and rotation speed of the dissolution apparatus on the PEO matrix erosion rate were studied to research the stability of these matrix systems. In addition, to find the nature of the polymer material which affects the water uptake rate, the swelling rate, and the erosion rate of matrices, the rheology of polymers in this system was also studied.

\section{The Study of Water Uptake, Swelling, and Erosion of PEO} Matrices

Each tablet which contains all excipients without drugs was placed in a beaker with $500 \mathrm{ml}$ of distilled water, and then measured by using a dissolution apparatus (ZRCD6-B) with the basket method (USP Apparatus I). The paddles were rotated at $50 \mathrm{rpm}$ at $37 \pm 0.5^{\circ} \mathrm{C}$. The tablets were taken out from the medium at different time intervals $(5,15,30$,

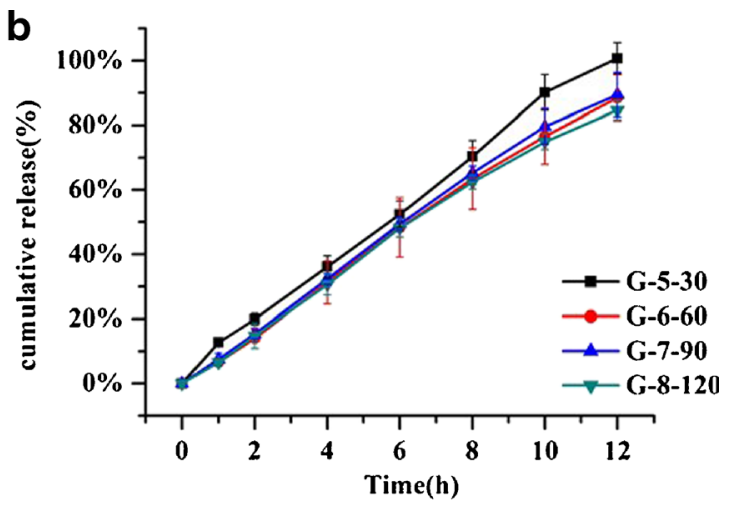

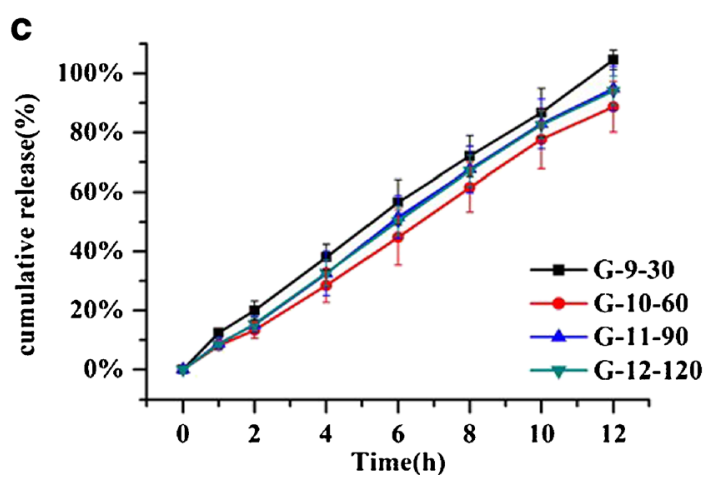

Fig. 1. The effects of dose of GLZ on drug release of three optimized PEO binary systems: WSRN 301 and PEON 80 (a); WSRN 301 and F68 (b); WSRN 301 and PEG 4000 (c) 

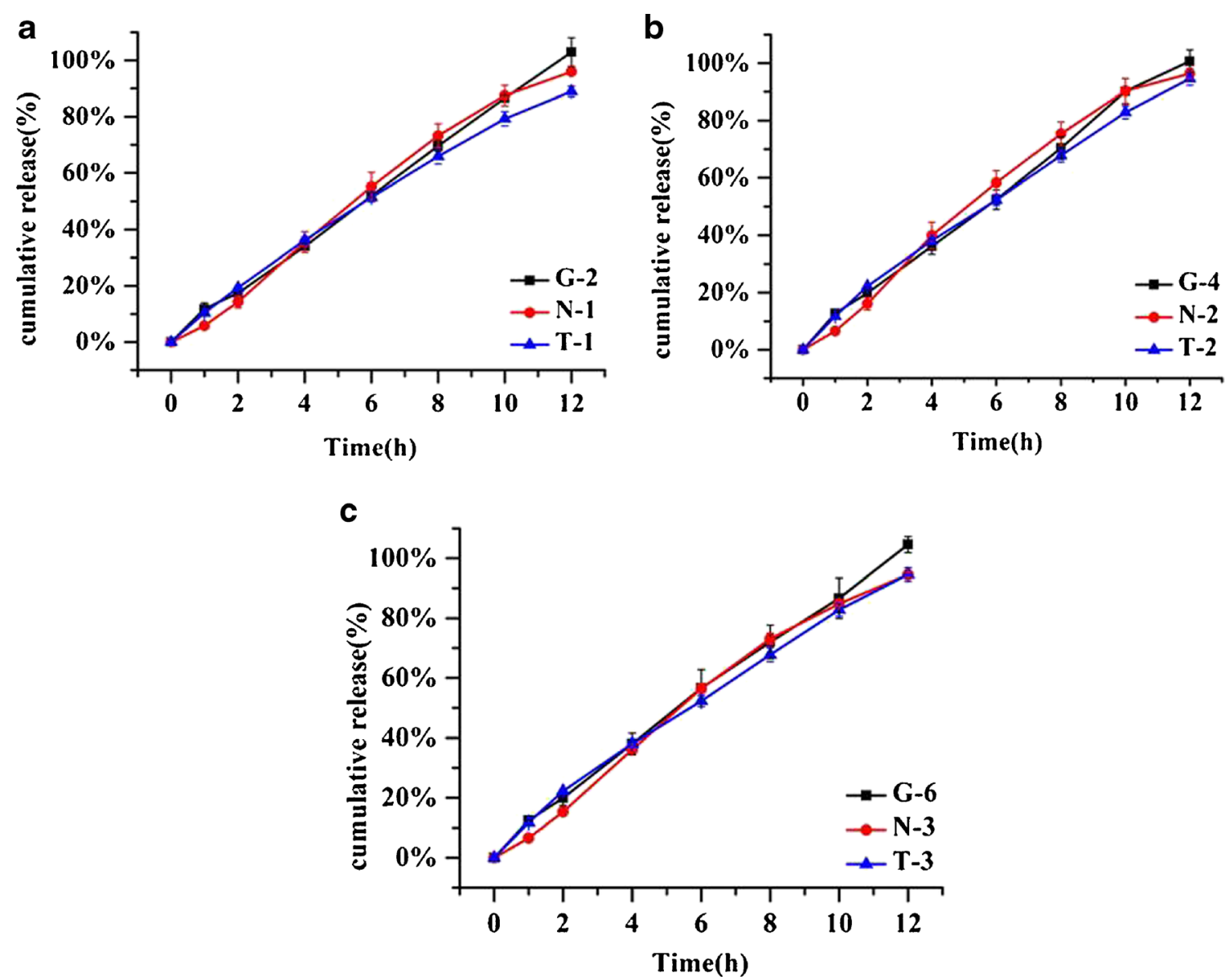

Fig. 2. The effects of different solubility of water insoluble drug on three optimized PEO binary systems release: WSRN 301 and PEON 80 (a); WSRN 301 and F68 (b); WSRN 301 and PEG 4000 (c)

$45,60,90,120,240,360$, and $480 \mathrm{~min})$ and removed the excess liquid and then weighed $\left(\mathrm{W}_{2}\right)$. And, dried in the drying oven for $48 \mathrm{~h}$ and weighed $\left(\mathrm{W}_{1}\right)$. The percentage of the water uptake was determined by using Eq. (5):

$\%$ water uptake $=\left(\mathrm{W}_{2}-\mathrm{W}_{1}\right) / \mathrm{W}_{1} \times 100$
At the end of the water uptake study, the swelling index (SI) was tested and calculated according to Eq. (3), and values of SI were recorded and then plotted against time on a graph. $\mathrm{W}_{0}$ is the initial tablet weight, and the swelling profiles were plotted by measuring tablets from three different batches at 1 , 2, 4, 6, 8, 10, and $12 \mathrm{~h}$.

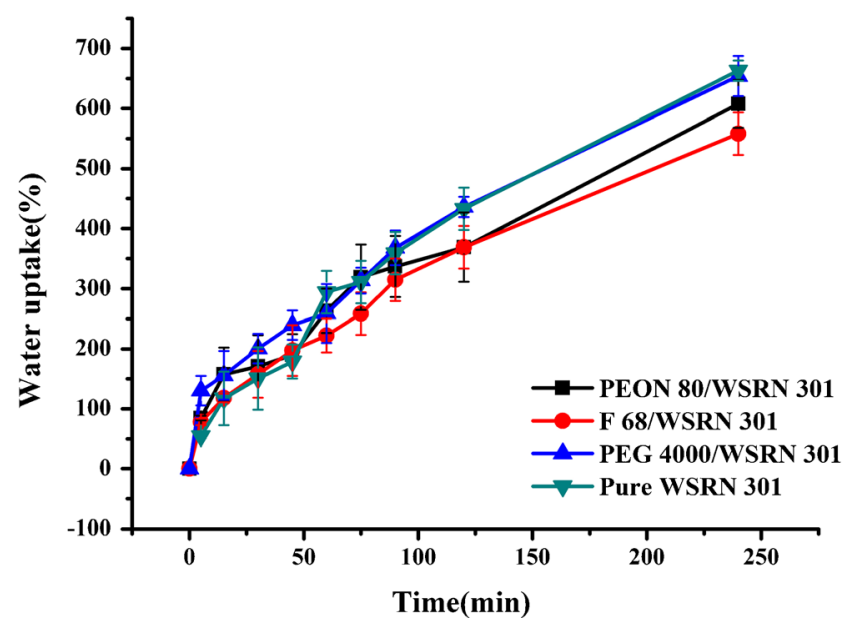

Fig. 3. Water uptake curve of the three optimized formulations and pure WSRN 301

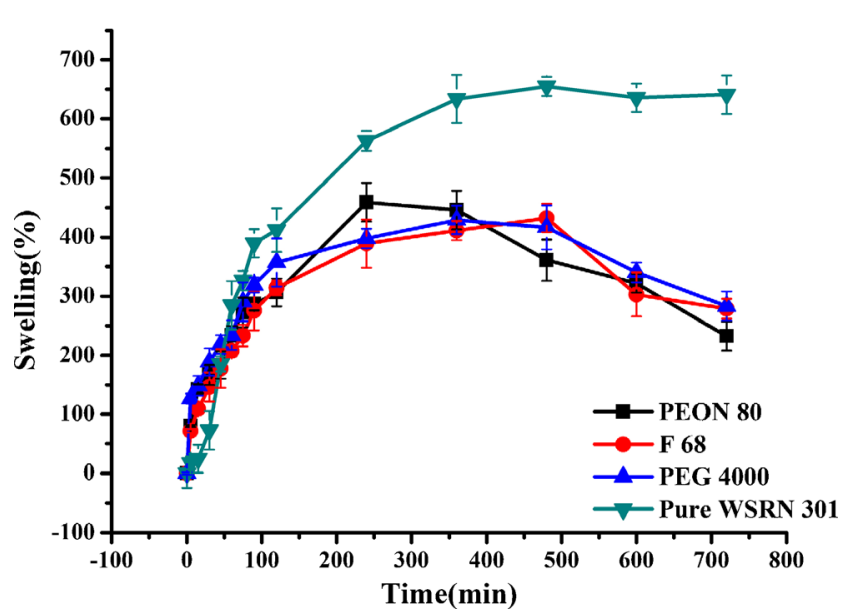

Fig. 4. Swelling curve of the three optimized formulations and pure WSRN 301 


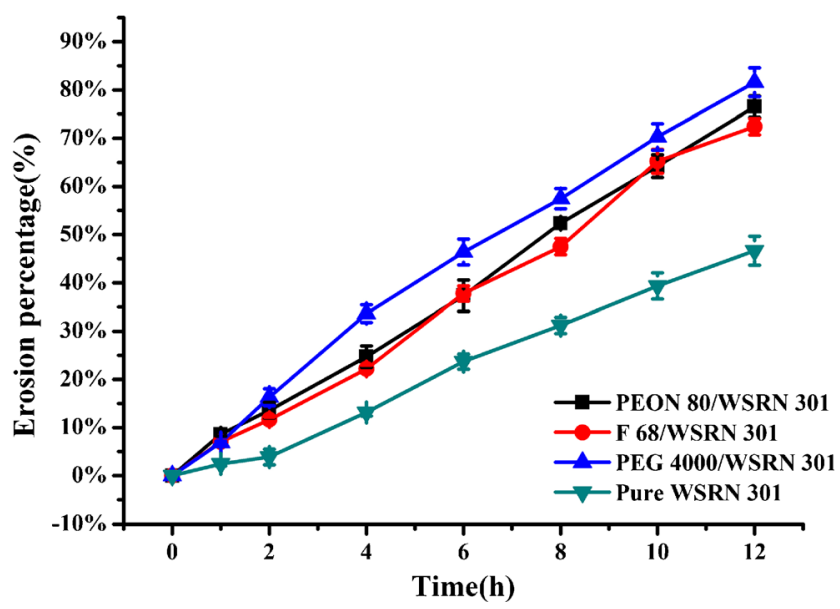

Fig. 5. Erosion curve of the three optimizing formulations and the pure WSRN 301

$\% \mathrm{SI}=\left(\mathrm{W}_{2}-\mathrm{W}_{0}\right) / \mathrm{W}_{0} \times 100$

At the end of each water uptake study, the erosion index (EI) was calculated using the following Eq. (4) and then plotted against time $(1,2,4,6,8,10,12 \mathrm{~h})$ in a graph:

$\% \mathrm{EI}=\left(\mathrm{W}_{0}-\mathrm{W}_{1}\right) / \mathrm{W}_{0} \times 100$

Study of the Effect of the $\mathrm{pH}$ and Rotation Speed on PEO Matrix Erosion Rate

The optimized binary matrix was used to investigate the effect of the dissolution medium on the PEO matrix erosion rate. In order to simulate the in vivo environment, the PEO binary matrix was run at $37 \pm 0.5^{\circ} \mathrm{C}$ using the following $500-\mathrm{ml}$ dissolution media and residence times: pH $1.2(0.1 \mathrm{~N} \mathrm{HCl}, 1.5 \mathrm{~h}) ; \mathrm{pH} 2.5$ (phosphate buffer, 1.5 h) pH 4.5 (phosphate buffer, 1.5 h); pH 7.0 (phosphate buffer, 3 h); pH 7.4 (phosphate buffer, 1 h) (27). Rotation speed was selected at $50 \mathrm{rpm}$. To evaluate the influence of the hydrodynamic conditions on the erosion rate, basket rotation speeds at 25, 50, 75, and $100 \mathrm{rpm}$ (28) were tested at the dissolution of $\mathrm{pH} 7.0$ and $37 \pm 0.5^{\circ} \mathrm{C}$. Cumulative erosion data were analyzed according to the zeroorder equation.

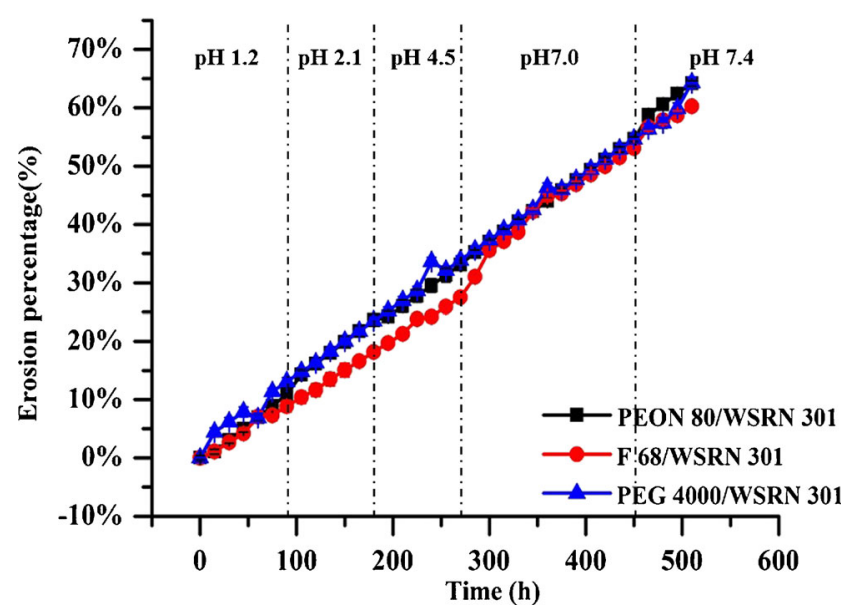

Fig. 6. The effects of different $\mathrm{pH}$ of dissolution medium on drug release from three optimized PEO binary systems

\section{Study Rheology of the Matrices}

As studied above, we investigated solution viscosities of several kinds of matrix system polymers to find the key influencing factors of the PEO-controlled water-insoluble drug release and guide us in finding the best combination. Flow curves and viscosities of the solution which were the matrices dissolved in releasing medium were studied by using a TA Instruments AR 2000 ex. The flow curves were obtained by the shear rate which increased from 0.1 to $120 \mathrm{~s}^{-1}$. This test was measured at $37 \pm 0.5^{\circ} \mathrm{C}$ in order to assess the body temperature.

\section{RESULTS}

\section{Study of GLZ Release From PEO Matrices and Release Kinetics}

As can be seen in Table IV, when the proportion of WSRN 301 and low viscosity hydrophilic materials was 1:1, the perfect dug release behavior was obtained. It was clearly observed that higher correlation coefficients of zero-order, first-order, and Korsmeyer-Peppas equations $\left(r^{2}>0.99\right)$ were acquired from the formulations G-2, G-4, and G-6 compared with formulations G-1, G-3, G-5, and G-7. And in order to obtain a good zero-order drug release behavior, the formulations G-2, G-4, and G-6 were selected as the optimized formulation to further study. Then, the similarity factor $f_{2}$ was

Table V. The Dissolution Kinetics of PEO Binary Matrix System

\begin{tabular}{llllr}
\hline Erosion zero-order kinetics & \multicolumn{2}{l}{ Matrix system } & & \\
\cline { 2 - 5 } & Pure WSRN 301 & $\begin{array}{c}\text { WSRN 301 and PEON } \\
80 \text { binary matrix }\end{array}$ & $\begin{array}{c}\text { WSRN 301 and F68 } \\
\text { binary matrix }\end{array}$ & $\begin{array}{r}\text { WSRN 301 and PEG } \\
4000 \text { binary matrix }\end{array}$ \\
\hline$r^{2}$ & 0.9971 & 0.9984 & 0.9949 & 0.9965 \\
$\mathrm{~T}_{50}(\mathrm{~h})$ & 21 & 13 & 13 & 12 \\
$K\left(\mathrm{~h}^{-1}\right)$ & 0.039 & 0.064 & 0.062 & 0.071 \\
\hline
\end{tabular}

$r^{2}$ correlation coefficient, $T_{50}$ time to erosion $50 \%$ of matrix, $K$ dissolution rate of constant 
Table VI. The Dissolution Kinetics of PEO Binary Matrix System in Different pH and Different Rotation Speed

\begin{tabular}{llcc}
\hline$r^{2}$ (zero-order) & \multicolumn{2}{l}{ Matrix system } & \\
\cline { 2 - 4 } & $\begin{array}{c}\text { WSRN 301 and PEON 80 binary } \\
\text { matrix }\end{array}$ & $\begin{array}{c}\text { WSRN } 301 \text { and F68 binary } \\
\text { matrix }\end{array}$ & $\begin{array}{c}\text { WSRN 301 and PEG } \\
\text { matrix }\end{array}$ \\
\hline$r^{2}($ simulate the in vivo & 0.9982 & 0.9924 & 0.9901 \\
pH) & 0.9714 & 0.9732 & 0.9862 \\
$r^{2}(25 \mathrm{rpm})$ & 0.9927 & 0.9947 & 0.9988 \\
$r^{2}(50 \mathrm{rpm})$ & 0.9913 & 0.9905 & 0.9960 \\
$r^{2}(75 \mathrm{rpm})$ & 0.9852 & 0.9863 & 0.9772 \\
$r^{2}(100 \mathrm{rpm})$ & & & \\
\hline
\end{tabular}

used to compare these three optimized formulations. As a result, there was no significant difference between them $\left(f_{2}>50\right)$. It demonstrated that these low-viscosity hydrophilic materials slightly influence the drug release rate. Table IV has shown that the $n$ values of these optimal formulations were higher than 0.89 and their correlation coefficients $\left(r^{2}\right)$ of zeroorder kinetic equation were higher than 0.99 , which indicated that they all acquired good controlled release.

\section{The Applicability of the PEO Matrix System}

In order to study the range of PEO matrices' drug loading, we studied the dose of GLZ in the matrices. And, the in vitro release profiles of $30,60,90$, and 120 -mg matrices were investigated. As it can be seen in Fig. 1, these formulations' drug release profiles have no significant difference $\left(f_{2}>50\right)$. The kinetic parameters of these formulations all showed a good zero-order $\left(r^{2}>0.99\right)$.
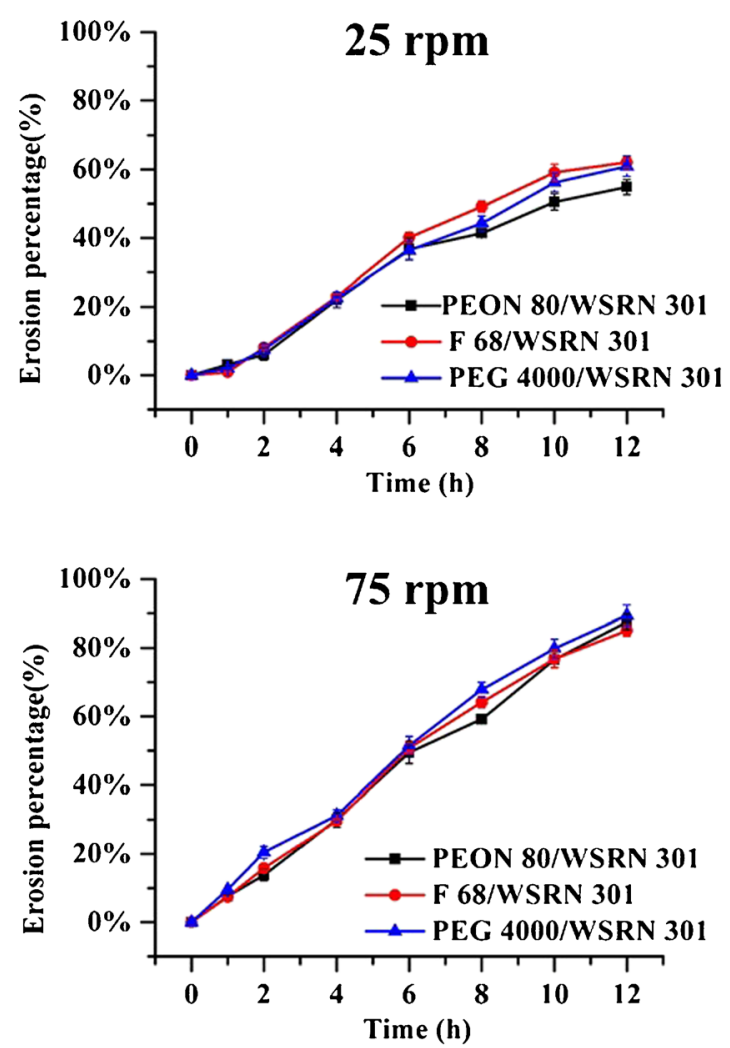

Therefore, the GLZ loading of PEO matrices was from 10.98 to $43.95 \%$. Based on this drug loading, we prepared the $60-\mathrm{mg}$ NIM and the 100-mg TPL matrix tablets to study the range of the drug solubility in these PEO matrix systems. As shown in Fig. 2, the three optimized formulations were all able to control NIM and TPL release with zero-order kinetics. In other words, these three PEO binary matrix systems can hold the water-insoluble drug solubility from several micrograms $(\mu \mathrm{g} / \mathrm{ml})$ to several milligrams $(\mathrm{mg} / \mathrm{ml})$. Of course, this solubility range includes GLZ (29).

\section{The Characteristics of These Three PEO Matrix Systems}

Water Uptake Behavior

The study of the water absorption was performed to further discuss the swelling behavior and erosion rate of PEO tablets. The results (in Fig. 3) showed that the hydro-
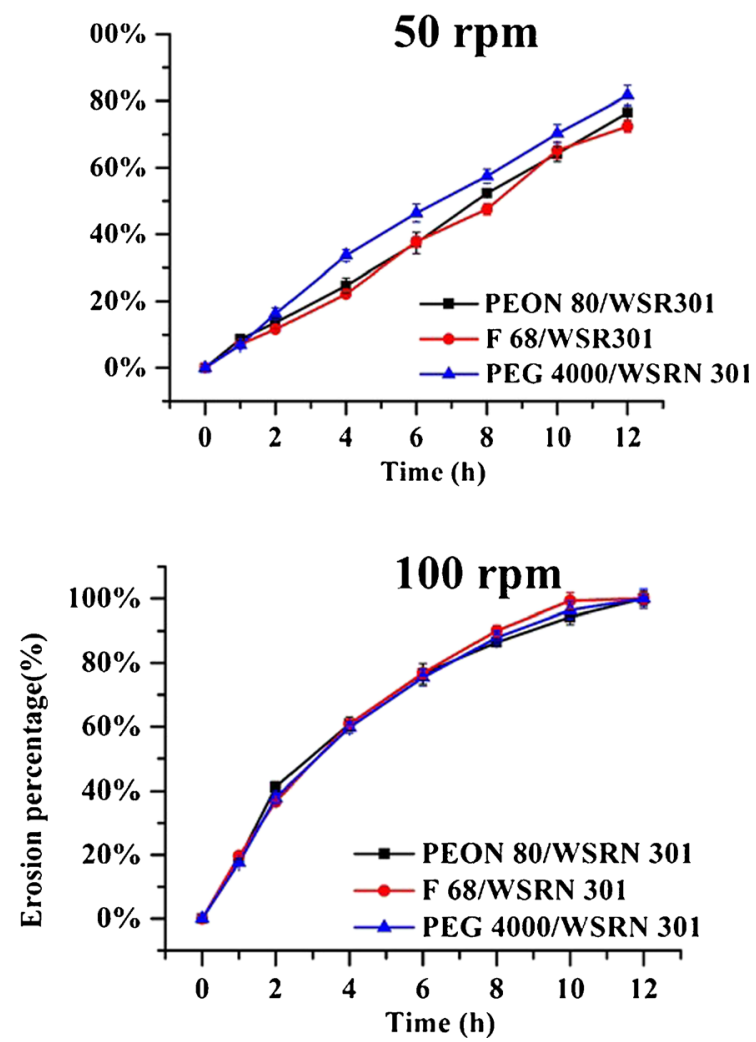

Fig. 7. The effects of different rotation speed on drug release from the three optimized PEO binary systems 
Table VII. The Effect of Different Rotation Speeds on Erosion Rate of PEO Binary Systems

\begin{tabular}{lllllll}
\hline$f_{2}$ & \multicolumn{3}{l}{ Rotation speed } \\
\cline { 2 - 7 } & 25 vs 50 & 25 vs 75 & 25 vs 100 & 50 vs 75 & 50 vs 100 & 75 vs 100 \\
\hline WSRN 301 and PEON 80 binary matrix & 49 & 38 & 29 & 49 & 35 & 41 \\
WSRN 301 and F68 binary matrix & 49 & 44 & 32 & 40 & 39 & 44 \\
WSRN 301 and PEG 4000 binary matrix & 46 & 37 & 32 & 39 & 42 \\
\hline
\end{tabular}

philic materials of low viscosity exhibit higher water uptake than pure PEO (WSRN 301), and this resulted in a rapid increase in the weight. In the first $90 \mathrm{~min}$, these matrices showed a high water uptake rate, and there were no significant differences between the three PEO binary systems except pure WSRN 301.

\section{Swelling Behavior}

As can be seen from Fig. 4, pure PEO was bigger than the swelling of the other three optimized matrix tablets. This could be explained that when the low viscosity polymer is added in the PEO matrix system, the interaction force of PEO (WSRN 301) chains would become weak after that matrix tablets dissolves. Therefore, as gel strength decreased, little swelling behavior exhibited on the surface of these PEO binary matrices.
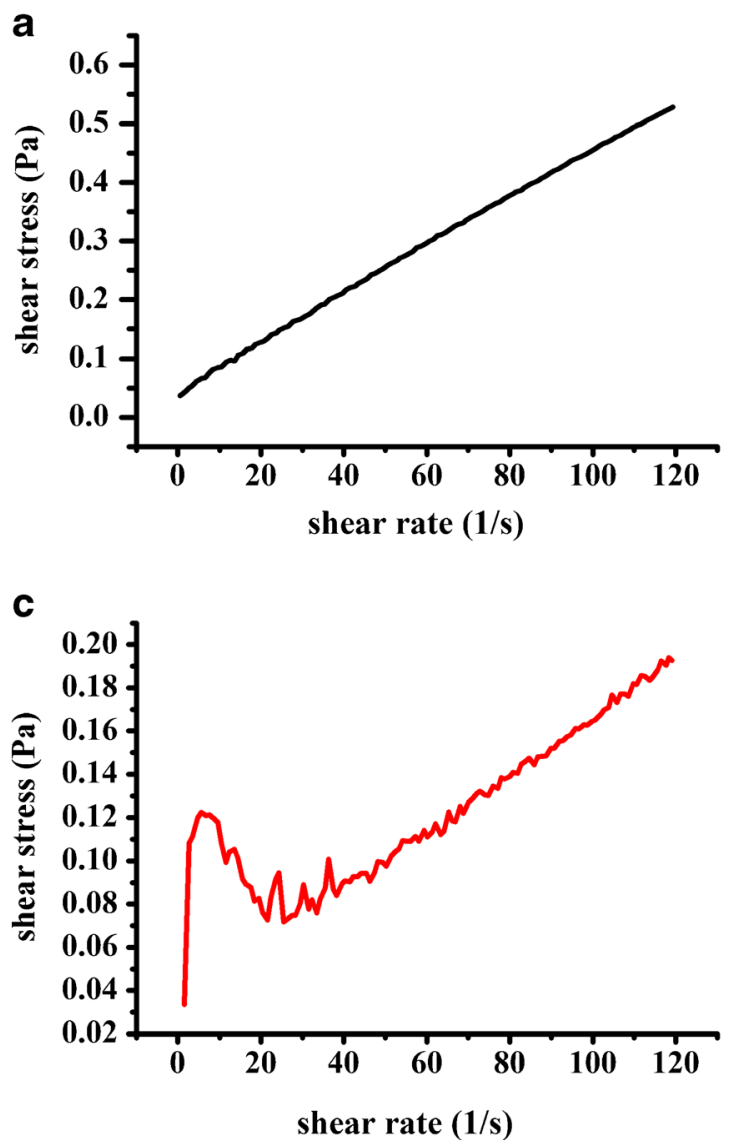

\section{Erosion Behavior}

As can be seen from Fig. 5, the erosion rate of pure WSRN 301 was very slow, the dissolution amount at $12 \mathrm{~h}$ was only $50 \%$, and $\mathrm{T}_{50}$ of zero-order was $21 \mathrm{~h}$ (in Table V), although the plot showed a zero-order kinetics $\left(r^{2}>0.99\right)$. However, when WSRN 301 combined with hydrophilic materials of low viscosity, the dissolution curve not only had zero-order behavior but also dissolution amount at $12 \mathrm{~h}$ was more than $75 \%$ (in Fig. 5), and the time of matrix erosion of $50 \%$ was about $13 \mathrm{~h}$. Because these three binary matrix systems had good zero-order kinetics and fast release rate, they can provide a favorable condition to control the insoluble drugs as zero-order release. We can design poorly soluble drug release behavior by preparing these binary matrices based on the characteristics of the PEO binary matrix system.

b

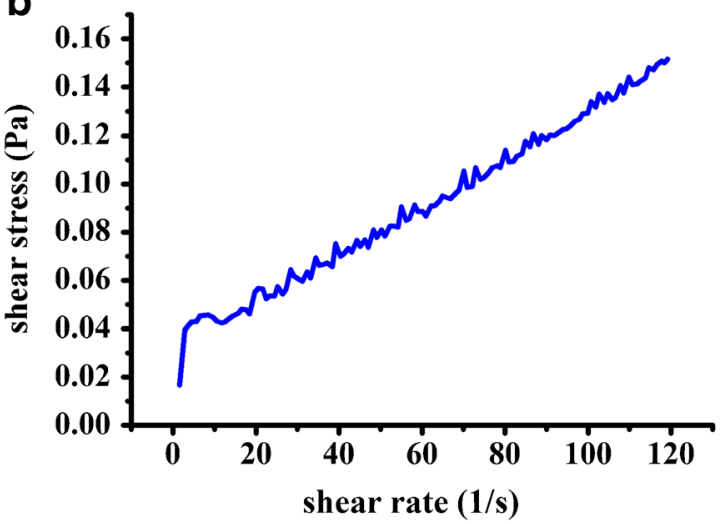

d

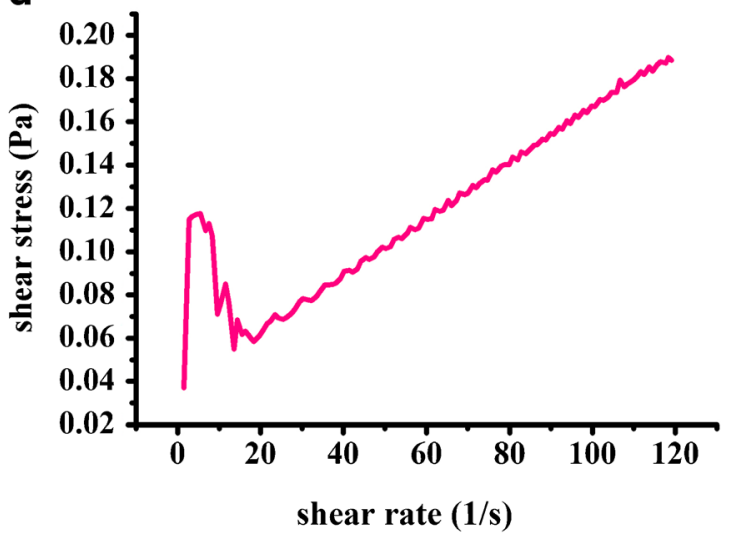

Fig. 8. The rheology curve of four hydrophilic polymers, pure WSRN 301 (a); pure PEON 80 (b); pure F68 (c); pure PEG 4000 (d) 
Table VIII. The Rheology of Materials

\begin{tabular}{|c|c|c|c|c|c|c|c|}
\hline & \multicolumn{4}{|c|}{ Pure materials } & \multicolumn{3}{|l|}{ Mixed materials } \\
\hline & $\begin{array}{l}\text { W S R N } \\
301\end{array}$ & PEON 80 & F68 & PEG 4000 & $\begin{array}{l}\text { WSRN 301/PEON } \\
\quad 80\end{array}$ & $\begin{array}{l}\text { WSRN 301/ } \\
\text { F68 }\end{array}$ & $\begin{array}{l}\text { WSRN } 301 / \text { PEG } \\
\quad 4000\end{array}$ \\
\hline $\begin{array}{l}\text { Concentration ( } \mu \mathrm{g} / \\
\quad \mathrm{ml})\end{array}$ & 0.12 & 0.12 & 0.12 & 0.12 & $0.6 / 0.6$ & $0.6 / 0.6$ & $0.6 / 0.6$ \\
\hline Apparent viscosity & $5.99 \mathrm{E}-03$ & $4.54 \mathrm{E}-04$ & $3.14 \mathrm{E}-04$ & $6.09 \mathrm{E}-05$ & $6.57 \mathrm{E}-03$ & $5.13 \mathrm{E}-03$ & $1.38 \mathrm{E}-03$ \\
\hline$S D$ & 2.742 & 14.85 & 14.03 & 17.7 & 14.74 & 8.616 & 6.179 \\
\hline
\end{tabular}

\section{The Effect of pH and Rotation Speed on Matrix Erosion Rates}

Characteristics of the PEO binary matrices showed us that the mechanism to control insoluble drug release was the erosion behavior of the binary matrices. And, the different erosion behaviors would produce a different in vitro drug release profiles. The matrix tablet erosion played a more important role than water uptake in the whole process of drug release.

Figure 6 indicated that when the $\mathrm{pH}$ of dissolution medium changed from 1.2 to 7.4 , the matrix erosion behavior had no significant difference $\left(r^{2}>0.99\right.$, good linear in Table VI). That is to say, these PEO binary matrix system erosion rates were not affected by $\mathrm{pH}$ of the dissolution medium. However, Fig. $7\left(\mathrm{r}^{2}<0.99\right)$ and Table VII $\left(f_{2}<50\right)$ suggested that PEO binary matrix erosion was influenced by the rotation speed, and this result was in accordance with the characteristics of hydrophilic matrix tablets which several authors have examined $(30,31)$.

\section{The Rheology}

Along with the water entering the matrix, the gel layer gradually formed. Because of the higher viscosity of the polymer, the stronger gel layer would be formed. This would prevent polymer erosion and then the drug release rate would become slow. The polymer dissolving rate was dependent on their chemical structure, molecular weight, the interactions with dissolution medium, and the gel layer strength. As a linear polymer, such as PEO, its molecular weight and viscosity had a significant effect on the drug release rate. When the pure PEO was unable to control drug release very well, the other kinds of hydrophilic materials were needed to add in prescriptions. Figure 8 shows us that even though these four pure materials all fitted the equation of the Herschel-Bulkley model (32) $(\mathrm{SD}<20$, the value of $\mathrm{SD}$ was usually used to evaluate the fitting degree of rheology equation and this value less than 20 was regarded as fitting very well), their viscosities was different. Table VIII suggests that adding the lowviscosity hydrophilic materials to WSRN 301 would reduce the viscosity of WSRN 301. Then, these three PEO binary matrix systems would not only exhibit three similar flow patterns but also provide a similar apparent viscosity. With the increase of shear rate, the share stress trend of these PEO binary matrices was very similar (in Fig. 9) and was changing within the same magnitude. If similar patterns and viscosities of PEO binary matrix solution were shown, the similar PEO matrix erosion and in vitro water-insoluble drug would be observed.

\section{DISCUSSION}

Hydrophilic matrices are the most famous DDS for the controlled drug release. There are many factors that influence the drug release from hydrophilic matrices, including drug solubility, types of hydrophilic materials, and proportion of these hydrophilic materials in the matrix systems (33). Waterinsoluble drugs (GLZ, NIM, and TPL) with different solubilities have been used to prepare the PEO binary matrix systems which include low-viscosity hydrophilic materials (PEON 80, F68, PEG 4000). The results have shown that when the drug solubility is within a certain range, the most influencing factor of the drug release rate is the viscosity of PEO binary matrix systems.

WSRN 301 has a high molecular weight and viscosity to form very strong gel layers, which would decrease the drug release rate $(34,35)$. On the contrary, if the polymer viscosity is reduced, the strength of the gel layer will become weak and the drug release rate will become fast. Forming a gel layer includes two steps: water uptake and swelling. These two steps take place at the whole process of drug release. When water absorption and swelling reach a critical value, the matrices start to dissolve and release the drugs (36). If the water absorption and polymer swelling cooperate very well, ideal drug release kinetics will be obtained. Therefore, by changing the viscosity of binary polymer in hydrophilic matrix tablets, a constant zero-order release rate could be obtained for waterinsoluble drugs.

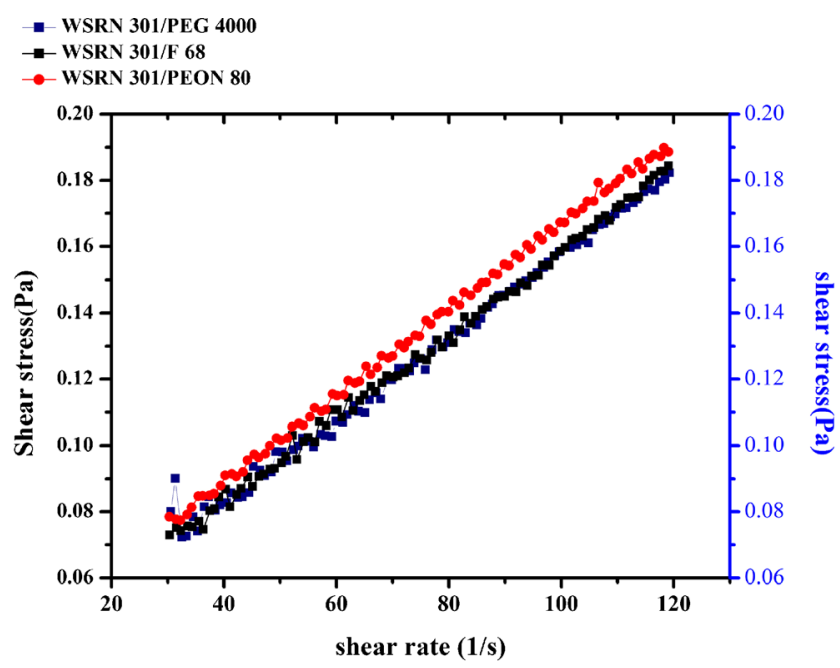

Fig. 9. The shear stress changes of these optimizing PEO binary systems when the shear rate from $30(1 / \mathrm{s})$ increases to $120(1 / \mathrm{s})$ 
The hydrophilic matrices, including WSRN 301 and the low-viscosity hydrophilic materials such as PEON 80, F68, and PEG 4000, produce a gel layer on the surface of the tablets. When the ratio of PEO and hydrophilic materials changes, the viscosity of the binary matrix will be changed, which would result in different erosion rates and drug release rates. Hydrophilic materials can change the drug release rate because their viscosity directly affects the strength of the gel layer and $C_{c r i t}$. In our studies, four hydrophilic polymers have different viscosities. However, the binary matrix system containing the same proportion of WSRN 301 and these low viscosity polymers showed same pattern of rheology and similar viscosity. Therefore, viscosity of hydrophilic systems played a key role in controlling drug release rate in this binary matrix system for water-insoluble drugs, which help us to design the hydrophilic matrix formulations.

The critical concentration also plays a very important role in hydroxypropyl methylcellulose (HPMC) matrix system dissolution (13). Moreover, the drug release from the HPMC matrix system is also affected by the substituted heterogeneity of HPMC. The substituted heterogeneity facilitated hydrophobic interactions to increase viscosity and therefore changed the gel layer strength and drug release rate. Thus, both molecular weight and substituted heterogeneity should be considered in HPMC matrix formulation design, while for PEO formulation, one should only consider the molecular weight.

\section{CONCLUSIONS}

Binary PEO hydrophilic matrix systems with other hydrophilic materials were developed to zero-order controlled release of water-insoluble drug with the solubility range from 2.27 to $\sim 8.3 \mathrm{mg} / \mathrm{ml}$. The controlled drug release rate was mainly determined by the viscosity of the hydrophilic systems. System viscosity is the most important factor to control drug release in the hydrophilic matrix system. Our research provides an effective guidance to choose appropriate formulations for hydrophilic matrix tablets, which brings benefit to reduce trial-and-error in formulation design.

\section{COMPLIANCE WITH ETHICAL STANDARDS}

\section{Conflict of interest conflict of interest.}

The authors report that they have no

\section{REFERENCES}

1. Zaid AN. Attitude and perception of patients and health care practitioners toward oral sustained release dosage forms in Palestine. Saudi Pharm J. 2010;18(4):251-6.

2. Claeys B, Vervaeck A, Hillewaere XKD, Possemiers S, Hansen $\mathrm{L}$, De Beer $\mathrm{T}$, et al. Thermoplastic polyurethanes for the manufacturing of highly dosed oral sustained release matrices via hot melt extrusion and injection molding. Eur J Pharm Biopharm. 2015;90:44-52.

3. Huang X, Brazel CS. On the importance and mechanisms of burst release in matrix-controlled drug delivery systems. J Control Release. 2001;73(2-3):121-36.
4. Aguilar-de-Leyva Á, Cifuentes C, Rajabi-Siahboomi AR, Caraballo I. Study of the critical points and the role of the pores and viscosity in carbamazepine hydrophilic matrix tablets. Eur J Pharm Biopharm. 2012;80(1):136-42.

5. Chopra S, Patil GV, Motwani SK. Release modulating hydrophilic matrix systems of losartan potassium: optimization of formulation using statistical experimental design. Eur J Pharm Biopharm. 2007;66(1):73-82.

6. Borgquist P, Körner A, Piculell L, Larsson A, Axelsson A. A model for the drug release from a polymer matrix tablet—effects of swelling and dissolution. J Control Release. 2006;113(3):21625.

7. Huanbutta K, Cheewatanakornkool K, Terada K, Nunthanid J, Sriamornsak P. Impact of salt form and molecular weight of chitosan on swelling and drug release from chitosan matrix tablets. Carbohydr Polym. 2013;97(1):26-33.

8. Caccavo D, Cascone S, Lamberti G, Barba AA. Controlled drug release from hydrogel-based matrices: experiments and modeling. Int J Pharm. 2015;486(1-2):144-52.

9. Jain AK, Söderlind E, Viridén A, Schug B, Abrahamsson B, Knopke C, et al. The influence of hydroxypropyl methylcellulose (HPMC) molecular weight, concentration and effect of food on in vivo erosion behavior of HPMC matrix tablets. J Control Release. 2014;187:50-8.

10. Sako K, Sawada T, Nakashima H, Yokohama S, Sonobe T. Influence of water soluble fillers in hydroxypropyl methylcellulose matrices on in vitro and in vivo drug release. J Control Release. 2002;81(1-2):165-72.

11. Bettini R, Catellani PL, Santi P, Massimo G, Peppas NA, Colombo P. Translocation of drug particles in HPMC matrix gel layer: effect of drug solubility and influence on release rate. J Control Release. 2001;70(3):383-91.

12. Campos-Aldrete ME, Villafuerte-Robles L. Influence of the viscosity grade and the particle size of HPMC on metronidazole release from matrix tablets. Eur J Pharm Biopharm. 1997;43(2):173-8.

13. Viridén A, Larsson A, Wittgren B. The effect of substitution pattern of HPMC on polymer release from matrix tablets. Int J Pharm. 2010;389(1-2):147-56.

14. Gupta CR, Kishore GK, Ratna JV. Development and evaluation of aceclofenac matrix tablets using polyethylene oxides as sustained release polymers. J Pharm Res. 2013;6(2):249-54.

15. Ishikawa T, Watanabe Y, Takayama K, Endo H, Matsumoto M. Effect of hydroxypropyl methylcellulose (HPMC) on the release profiles and bioavailability of a poorly water-soluble drug from tablets prepared using macrogol and HPMC. Int J Pharm. 2000;202(1-2):173-8.

16. Jamzad S, Tutunji L, Fassihi R. Analysis of macromolecular changes and drug release from hydrophilic matrix systems. Int J Pharm. 2005;292(1-2):75-85.

17. Katakam P, Padala NR, Chandu BR, Elfituri A, Adiki SK, Kommu R. Design of lamivudine XR matrix tablets: Influence of HPMC and PEO on in vitro drug release and bioavailability in rabbits. J Pharm Res. 2013;6(8):845-52.

18. Park JS, Shim JY, Truong NKV, Park JS, Shin S, Choi YW, et al. A pharma-robust design method to investigate the effect of PEG and PEO on matrix tablets. Int J Pharm. 2010;393(1-2):80-8.

19. Kojima H, Yoshihara K, Sawada T, Kondo H, Sako K. Extended release of a large amount of highly water-soluble diltiazem hydrochloride by utilizing counter polymer in polyethylene oxides (PEO)/polyethylene glycol (PEG) matrix tablets. Eur J Pharm Biopharm. 2008;70(2):556-62.

20. Maggi L, Segale L, Torre ML, Ochoa Machiste E, Conte U. Dissolution behaviour of hydrophilic matrix tablets containing two different polyethylene oxides (PEOs) for the controlled release of a water-soluble drug. Dimensionality study. Biomaterials. 2002;23(4):1113-9.

21. Korsmeyer RW, Peppas NA. Effect of the morphology of hydrophilic polymeric matrices on the diffusion and release of water soluble drugs. J Membr Sci. 1981;9(3):211-27.

22. Costa P. An alternative method to the evaluation of similarity factor in dissolution testing. Int J Pharm. 2001;220(1-2):77-83.

23. Xie F, Ji S, Cheng Z. In vitro dissolution similarity factor (f2) and in vivo bioequivalence criteria, how and when do they match? 
Using a BCS class II drug as a simulation example. Eur J Pharm Sci. 2015;66:163-72.

24. Huang S, Yu X, Yang L, Song F, Chen G, Lv Z, et al. The efficacy of nimodipine drug delivery using mPEG-PLA micelles and mPEGPLA/TPGS mixed micelles. Eur J Pharm Sci. 2014;63:187-98.

25. Yoon HS, Lee JH, Lim ST. Utilization of retrograded waxy maize starch gels as tablet matrix for controlled release of theophylline. Carbohydr Polym. 2009;76(3):449-53.

26. Riekes MK, Caon T, da Silva JJ, Sordi R, Kuminek G, Bernardi LS, et al. Enhanced hypotensive effect of nimodipine solid dispersions produced by supercritical $\mathrm{CO} 2$ drying. Powder Technol. 2015;278:204-10.

27. Ashford M. The gastrointestinal tract-physiology and drug absorption. In: Aulton ME, editor. Pharmaceutics: the science of dosage form design. Edinburgh.: Churchill Livingstone; 2002. p. 217-33.

28. Katori N, Aoyagi N, Terao T. Estimation of agitation intensity in the GI tract in humans and dogs based on in vitro/in vivo correlation. Pharm Res. 1995;12(2):237-43.

29. Hong SS, Lee SH, Lee YJ, Chung SJ, Lee MH, Shim CK. Accelerated oral absorption of gliclazide in human subjects from a soft gelatin capsule containing a PEG 400 suspension of gliclazide. J Control Release. 1998;51(2-3):185-92.
30. Morihara M, Aoyagi N, Kaniwa N, Katori N, Kojim S. Hydrodynamic flows around tablets in different pharmacopeial dissolution tests. Drug Dev Ind Pharm. 2002;28(6):655-62.

31. Wu Y, Ghaly ES. Effect of hydrodynamic environment on tablet dissolution using flow-through dissolution apparatus. P R Health Sci J. 2006;25(1):75-83.

32. Mullineux G. Non-linear least squares fitting of coefficients in the Herschel-Bulkley model. Appl Math Model. 2008;32(12):253851.

33. Maderuelo C, Zarzuelo A, Lanao JM. Critical factors in the release of drugs from sustained release hydrophilic matrices. J Control Release. 2011;154(1):2-19.

34. Viridén A, Wittgren B, Larsson A. Investigation of critical polymer properties for polymer release and swelling of HPMC matrix tablets. Eur J Pharm Sci. 2009;36(2-3):297-309.

35. Kavanagh N, Corrigan OI. Swelling and erosion properties of hydroxypropyl methylcellulose (Hypromellose) matrices-influence of agitation rate and dissolution medium composition. Int J Pharm. 2004;279(1-2):141-52.

36. Viridén A, Wittgren B, Andersson T, Larsson A. The effect of chemical heterogeneity of HPMC on polymer release from matrix tablets. Eur J Pharm Sci. 2009;36(4-5):392-400. 